\title{
LE SON DE RIZ DANS L'ALIMENTATION DES VACHES LAITIERES
}

\author{
par \\ Telesforo BONADONNA \\ Directeur de la Société des Eleveurs de la Province de Pavie.
}

\section{BUTS ET MOYENS DES REGHERGHES}

Le problème de l'utilisation du son de riz dans l'alimentation du bétail domestique n'est pas du tout nouveau. En effet, ce produit peut être administré à presque toutes les espèces d'animaux. Dans les régions rizières, le son est particulièrement utilisé pour l'alimentation des vaches laitières, des bovins en général, des pores, et de tous les animaux de travail.

Cependant, l'emploi du son de riz comme nourriture des vàches laitières est encore assez controversé. Les plus acharnés contre ce système d'alimentation sont les industriels du lait, qui soutiennent que des rations trop copieuses de son de riz causent des insuccès considérables dans le traitement industriel du lait, c'est-à-dire bouffissure et mauvaise conservation des fromages, altération des acides gras du beurre, etc.

D'autre part, la mise en valeur du son de riz est un problème technique et économique de la plus haute importance. L'industrie rizière italienne produit en effet près de 500.000 quintaux de son de riz, et les industries du riz sont étroitement liées aux intérêts des principales régions agricoles, ainsi qu'à céux de la nation tout entière. Celle-ci, en effet, accorde aux provinces des subventions qui atteignent plusieurs millions de lires par an. On pourra done aisément comprendre que la mise en valeur du son de riz, tant au, point de vue technique qu'au point de vue économique, représente, dans la pratique, une question essentielle d'ordre national.

C'est précisément à cause de ces problèmes que la Société des Eleveurs de Pavie (S. A. B. L.) a commencé, suivant des plans préfixés, une série de recherches, dans le but d'établir définitivement l'existence et l'importance des effets nuisibles présumés ou réellement tels, par rapport à la qualité et à la quantité administrées au bétail. Cette même Société se propose ainsi de rechercher les causes de ces inconvénients, pour découvrir les moyens propres à les faire disparaître, ou tout au moins, à les diminuer.

Le problème de l'utilisation alimentaire du son de riz atteint la plus grande importance dans la région de Pavie, où la culture intensive (presque un tiers de l'entière production nationale) est jointe à l'élevage des vaches laitières. En effet, cette région, outre 
son beurre très renommé, produit des fromages durs appréciés, du type "Grana ", "Reggio ", et " Gorgonzola ".

\section{Le son de riz}

Le son de riz est le plus important sous-produit du traitement industriel du riz et celui ayant la plus grande valeur zootechnique. Les nombreuses manipulations auxquelles le riz est soumis, visent à un double but : lui permettre une plus longue conservation, en le rendant, en même temps, commercialement plus agréable. La première opération consiste à décortiquer le gros riz. Les grains sont ainsi dégagés des glumelles qui les enveloppent. Les déchets, ou balle, qui en dérivent, n'ont aucune valeur zootechnique, parce qu'elles sont trop riches en cellulose, silex, etc. On polit ensuite le riz avec des machines spéciales à cône ou à hélice.

On appelle le riz ainsi traité, riz marchand. Ce riz a été plus ou moins privé des couches superficielles du péricarpe, et sa richesse en matières albuminoïdes, diminue la durée de conservation du produit. C'est justement pendant la phase industrielle du polissage qu'on produit le son de riz. Les traitements successifs auxquels le riz peut encore être soumis, ont lieu au contraire pour polir davantage, à l'aide de différentes substances (huile, talc, glucose) la surface des grains, pour leur donner l'aspect caractéristique du riz "Camolino » et du riz glacé. Parmi les sous-produits du riz, il y a le farinet de riz, dont l'importance alimentaire est remarquable à cause de sa richesse en amidon et parce qu'il contient encore des albuminoïdes et une petite quantité de matière grasse. Le plan suivant donnera une idée complète du cycle industriel pour le traitement du riz.

Somme toute, pour chaque quintal de gros riz, on obtient à peu près 17 kilogrammes de balle, de 6 à 7 kilogrammes de son de riz, et presque 1 kilogramme de farinet de riz.

On sépare le germe, ou embryon du riz, du son à l'aide de puissants ventilateurs. Le germe peut ainsi servir pour l'extraction de l'huile. Le son de riz tout à fait pur s'appelle commercialement vierge. Il a l'apparence d'une farine jaune rougeâtre, onctueuse et douce au toucher, d'odeur et de saveur douceâtre. Lorsque le son s'altère à cause de son âge ou de sa mauvaise conservation, il devient subitement aigre. La valeur alimentaire du son vierge est remarquable. Il contient en effet de 10 à $13 \%$ de protéine, de 12 à $15 \%$ de matière grasse, $35 \%$ de matières extractives, et à peine de 7 à $9 \%$ de cellulose. Son pouvoir nutritif est pareillement élevé, et il peut être évalué, d'après M. GidLiani, à 92 unités fourragères ou unités orge. Le son vierge présente néanmoins l'inconvénient de se conserver assez peu longtemps, puisqu'il aigrit facilement à cause de la matière grasse qu'il contient. Un autre désavantage du son de riz 


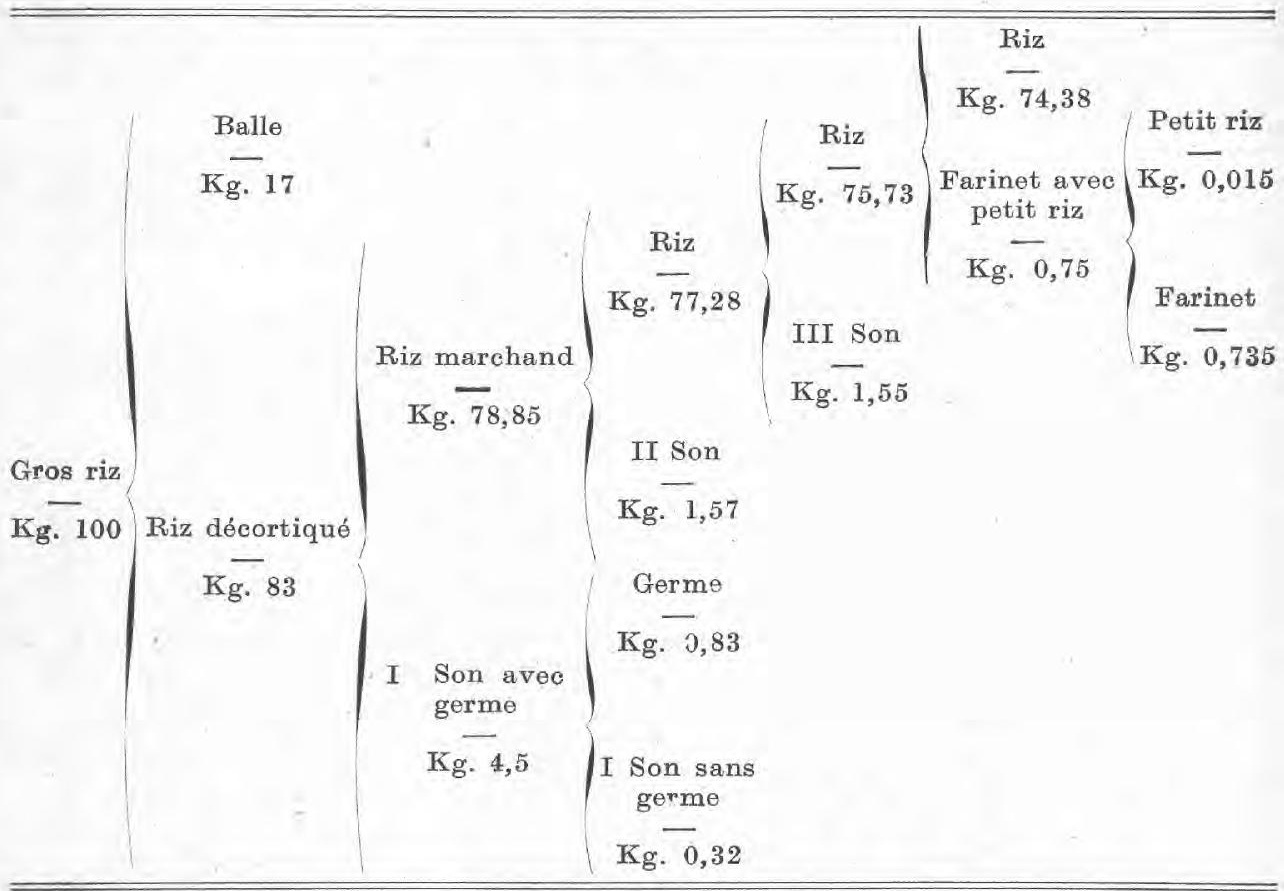

est que sa graisse est très riche en oléine. Pour en augmenter la conservation, mais surtout dans un but spéculatif, le son de riz est souvent sophistiqué par addition de plusieurs quantités de « fumetto » et de "tondello ». Ces deux produits proviennent de la mouture plus ou moins fine de la balle de riz. Naturellement, la présence de ces substances dans le son de riz, non seulement en diminue la valeur alimentaire, mais encore les petites écailles du «tondello», raboteuses et sileuses, peuvent être très nuisibles aux animaux, surtout par l'action irritante produite sur les premières voies respiratoires et par la toux consécutive (1).

Généralement sur le marché, le son de riz est classé et vendu en trois types différents, suivant le titre adipeux-protéique, et comme résultat de la somme des valeurs énoncées pour les deux éléments nutritifs, c'est-à-dire la protéine et les graisses (2) :

1. Son vierge à titre adipeux-protéique de 25 à $29 \%$.

2. Son $24 \%$ à titre adipeux-protéique de 23 à $24 \%$.

3. Son $18 \%$ à titre adipeux-protéique de 18 à $20 \%$.

(1) R, Givitani. Esperimenti d'alimentazione delle vacche da latte con la pula di riso, Milano, 1917 (Expériences d'alimentation des vaches laitières avec le son de riz, Milan, 1917).

(2) R. Giduiani, La pula di riso nell' alimentazione del bestiame, Firenze, 1935 (Le son de riz dans l'alimentation du bétail, Florence, 1935). 
Le troisième type de son, et plus encore les sons mélangés et davantage frelatés, sont en somme les moins propres à l'alimentation du bétail, même s'ils sont, comme on l'a déjà vu, les moins altérables. Dans une de ses récentes publications (1), M. GiUliani rapporte les données analytiques suivantes, sur les différents types de son de riz, indiquant, entre parenthèse, les limites tolérables d'oscillation:

\section{Son vierge \\ $\%$}

Eau

Protéines brutes

13,10

Matière grasse brute .

Matières extractives.

Cellulose .........

Cendres
$12,5(12-13)$

$15,4(13-16)$

$41,5(40-46)$

$10,2(8-10,5)$

$7,4(7-9)$
Son $24 \%$

9,1

$11,5(11-12)$

$12,5(11-13)$

$41,2(40-45)$

$14,1(11-15)$

$11,6(10-11,5)$
Son $18 \%$

10,7

$8,8(8-10,5)$
$9,5(9-10)$
$41,0(38-44)$
$18,2(16-20)$
$11,8(11-12)$

La facilité avec laquelle on frelate dans le commerce le son de riz, et sa courte conservation, sont les causes principales qui empêchent la diffusion de ce produit précieux, surtout dans les régions non rizières. D'ailleurs, il est certain que l'utilisation dans un but alimentaire du son altéré et en quelque manière imparfaite est un des motifs qui a rendu les industriels du lait particulièrement hostiles à l'emploi de cette sorte de fourrage, en raison des conséquences présumées ou réelles, qu'il entraîne pour les produits caséeux. Il s'ensuit que l'éleveur doit être très avisé, aussi bien en ce qui concerne les achats, que l'emmagasinage et la conservation du son de riz. On peut prolonger efficacement la conservation du son de riz. sans toutefois en altérer les qualités nutritives et "appétibles », en le soumettant pour une dizaine de minutes, à une température sèche de $60^{\circ}$. Le son de riz convenablement mélangé avec d'autres aliments dans les quantités nécessaires, peut être administré à toute espèce d'animaux domestiques, quelle qu'en soit leur destination économique. De nombreuses études expérimentales ont été faites à ce sujet, même en Italie, tant sur les chevaux que sur les pores, les ovins, les caprins, et les animaux de basse-cour. Les résultats obtenus ont toujours été satisfaisants et ils ont été aussi pleinement confirmés dans la pratique des élevages. L'emploi du son de riz comme aliment a été particulièrement étudié pour les bovins, et précisément pour les vaches laitières, dont l'élevage est florissant dans presque toutes les régions rizières italiennes. D'après les plus récentes études et suivant l'enseignement de la pratique, on recommande l'emploi du son de riz vierge et du son à $24 \%$, non pas seulement comme concentré exclusif, mais encore comme un ingrédient excellent et économique pour les mélanges complexes des tourteaux, farines, etc. Lorsqu'il s'agit de son vierge, on pourrait at-

(1) R. Giuliani. Voir le dernier traité cité. 
teindre sans inconvénients (d'après $M$. GIULIANI), même la proportion de 15 à $25 \%$. Ce même auteur croit que lorsque le lait est destiné à la fabrication des fromages durs à maturation lente, il est indiqué de limiter l'emploi du son de riz pour en éviter les inconvénients éventuels (bouffissures).

M. le Professeur Montr, directeur du Laboratoire provincial de Chimie, de la ville de Pavie (1), a dernièrement constaté, dans du beurre fabriqué avec le lait provenant des vaches alimentées avec du son de riz, une diminution des index des acides volatils solubles. Les données relatives aux expériences sur les différents régimes alimentaires, faites par le Professeur Monti, sont les suivantes:

\section{Acides volatils}

Solubles Insolubles A' $35^{\circ}$ (Zeiss)

Herbe, foin, son de blé ..............

29,2

2,3

45,6

Herbe, foin, tourteau de coco (1 kilogramme)

29,1

2,3

45,0

Herbe, foin, son de riz (2 kilogrammes) ....

25,9

1,5

47,0

Herbe, foin, tourteau d'arachide ........

28,9

2,2

45,8

Herbe, foin, son de riz (3 kilogrammes) ....

26,5

1,8

46,1

Herbe, foin, son de riz (après 6 jours) ......

19,3

1,2

48,5

Herbe, foin, son de riz (après 12 jours) .....

16,7

1,0

48,6

Le Professeur Montr, tout en annonçant la continuation de ses expériences, fait justement remarquer que, si le phénomène observé a une valeur tout à fait négligeable lorsqu'il s'agit de lait alimentaire, il peut devenir très important quand il s'agit de lait industriel, car les produits que l'on obtient du lait provenant des vaches alimentées avec du son de riz (beurre, fromages) n'offrent pas les qualités de pureté requises par la loi.

Le plan des recherches commencées par la S. A. B. L. renferme l'étude de tous les différents points de vue du problème de l'emploi alimentaire du son de riz, par rapport aux susdites destinations industrielles du lait.

On commence pourtant à apprécier le traitement du fromage dur "Grana », type "Reggio ", pour plusieurs raisons. En effet, les premières de ees recherches furent conduites dans un but d'orientation.

En poursuivant ses recherches, la S. A. B. L. a voulu tout d'abord adopter comme base de critère celle de maintenir inaltérées les conditions pratiques de production, de traitement et de conservation, de manière que toute cause de perturbation et de moindre véridicité des observations fût éliminée. Le problème en question a déjà été étudié ad hoc dans les Laboratoires et dans les Instituts, mais il

(1) N. Monri. Acides volatils du beurre provenant des vaches alimentées avec du son de riz. Le Lait, $n^{\circ} 146$, juin 1935. 
manque encore à sa résolution, cette base pratique suffisante pour rendre définitifs les résultats, et surtout agréables aux classes intéressées à la production et au traitement du lait.

La S. A. B. L., par rapport à cette ligne directive, croit être parmi les premières institutions qui aient transporté l'observation scientifique directement dans le champ administratif. Grâce à cet achèvement, la S. A. B. L., outre avoir certifié ses propres buts particuliers et statutaires, croit aussi avoir interprété de la meilleure façon les critères de la fondation Allocehio du Royal Institut Lombard de Sciences et Lettres.

\section{Critères poursuivis}

Nos recherches se sont naturellement conformées à la solution des problèmes alimentaires. A ces recherches on se propose d'établir la limite de l'utilité zoo-économique, pour l'emploi de différentes quantités de son de riz dans l'alimentation des vaches laitières, et d'établir les effets et l'action des différentes quantités de son de riz sur la réussite du traitement du fromage dur "Grana ", type "Reggio", fabriqué avec le lait provenant de ces mêmes vaches.

A cette intention, on a pris soin, au cours des recherches, de déterminer la qualité et la quantité des différents aliments concentrés ; de préciser la qualité et la quantité du lait obtenu; d'établir la nature et la modification de la flore bactérienne du lait, du petitlait employés, des fromages ; et de suivre les procédés de ces derniers pendant la première période de maturation (été), en attendant le développement des périodes successives.

Naturellement la S. A. B. L., profitant de la contribution qui lui fut accordée par la fondation Allocehio, en 1934, a dû pourvoir à :

a) Compléter l'équipement de son Laboratoire en le dotant d'un plus grand nombre d'appareils pour les recherches chimiques et pour les recherches bactériologiques.

b) Concourir aux frais d'alimentation des vaches, moyennant l'achat direct des aliments concentrés fondamentaux, à commencer par le son de riz, afin d'être sûr de leur qualité et de leur uniformité.

c) Indemniser le personnel de la ferme pour l'augmentation du travail et pour l'inciter à faciliter les expériences en cours. Rétribuer proportionnellement le personnel social chargé des mesurages, des contrôles sur place.

d) Couvrir les frais des contrôles analytiques exécutés par ordre de la Station de Chimie agraire de Milan et du Laboratoire provincial de Chimie de Pavie.

e) Liquider les frais de transport pour les vérifications effectuées sur place. 
L'expérience a eu lieu à la ferme de MM. SpIzzi Frères, à la "Cascina Cravino", dans la commune de Pavie. Cette ferme a une superficie de 91 hect. 63 . La rotation agraire adoptée pendant l'année 1933-1934 fut la suivante :

39 hect. 27 en prairies.

13 hect. 09 en prairies artificielles à arrosement hivernal. (Marcite).

13 hect. 74 en maïs.

7 hect. 20 en rizières.

18 hect. 32 en blé.

La vacherie, pendant la durée de l'expérience, se composait de 90 vaches et de 2 taureaux. La production quotidienne de lait était à peu près de 6 hectol. 5, équivalente donc à la quantité nécessaire pour la fabrication de deux pièces de fromage dur "Grana ", type "Reggio ", par jour. Le traitement industriel du lait était fait directement par les propriétaires fermiers à l'aide d'ouvriers spécialisés.

La technique normalement suivie dans l'alimentation des vaches et dans le traitement industriel du lait était, sinon parfaite, tout au moins des plus satisfaisantes. La conservation du fromage fabriqué, pendant un nombre variable de mois, se fait généralement dans les fromageries dont la ferme dispose. La remarquable habilité et la diligence des propriétaires fermiers a garanti l'excellente qualité des fourrages employés pendant toute la durée de l'expérience.

Voilà le plan, suivant lequel on a terminé les expériences :

1. Parmi les vaches de l'étable on en choisit 67. Elles furent ensuite distribuées en deux groupes homogènes quant au poids, au rendement, à l'état d'alimentation. Le premier groupe, ou groupe de contrôle, se composait de 34 animaux; le deuxième, ou groupe des expériences, de 33 animaux.

2. La durée entière de l'expérience qui était 65 jours, c'est-à-dire du 25 janvier au 30 mars 1935, fut partagée en cinq périodes :

a) Première période, ou période d'observation, de la durée de dix jours (25 janvier-2 février), pendant laquelle les animaux reçurent le même genre d'alimentation.

b) Deuxième période de quinze jours (4-16 février) pendant laquelle le groupe des expériences reçut un mélange de concentrés auxquels, outre les aliments en commun avec les vaches du groupe de contrôle, on ajouta le son de riz dans la quantité de $25 \%$.

c) Troisième période de quinze jours (18 février-4 mars) pendant laquelle la quantité de son de riz fut portée à $40 \%$.

d) Quatrième période de dix jours (6-15 mars) pendant laquelle la quantité du son fut réduite encore à $25 \%$. 
e) Cinquième période de quinze jours (16-30 mars) pendant laquelle on supprima l'administration du son de riz aux deux groupes, qui furent soumis au même régime alimentaire.

3. Les deux groupes furent partagés de manière que les animaux eussent à occuper les deux moitiés séparées de l'étable. Après avoir fixé les mélanges des concentrés, sous le contrôle direct de l'agent spécial de la S. A. B. L., le rationnement fut confié aux soins du chef d'étable pour ce qui concernait la quantité individuelle, tant des concentrés (en moyenne 2 kilogrammes pro die pour chaque animal), que des fourrages volumineux, et cela pour maintenir inaltérées, le plus possible, les conditions normales de la ferme.

4. Des domestiques de ferme chargés de la traite des vaches furent assignés aux deux groupes séparément. Le lait obtenu fut placé dans de grands seaux distincts, et transporté séparément à la fromagerie. Là on le transvasa dans de différents bassins d'affleurement, de manière que l'on put avoir la certitude que les deux pièces de fromage dur fussent fabriquées l'une avec le lait provenant des vaches du groupe de contrôle, et l'autre avec le lait provenant des vaches du groupe des expériences. Le petit-lait employé dans la pratique industrielle fut aussi soigneusement conservé en deux dames-jeannes distinctes.

5. La pesée des fourrages volumineux, des aliments concentrés, du lait provenant de chaque vache, était fait tous les deux jours. La détermination du contenu de matière grasse dans le lait était faite tour à tour, une fois pour le groupe des expériences, et une fois pour celui de contrôle. Tous les deux jours on déterminait l'acidité du petit-lait-levain. Le lait, le petit-lait-levain, le mélange de lait et petit-lait, étaient échantillonnés pour les recherches bactériologiques, et on tenait compte de la quantité de présure employée.

6. Chaque semaine on contrôlait les pièces de fromage, tout en effectuant des prélèvements supplémentaires de petit-lait-levain, de lait et petit-lait, de fourrages, de bouse fraîchement évacuée par les animaux des deux groupes; prélèvements destinés aux recherches bactériologiques.

7. Les aliments concentrés et le son de riz, fournis par la Société Agraire de Pavie, se trouvèrent toujours uniformes et de bonne qualité. Les analyses de ces mêmes produits furent contrôlées par la Station de Chimie Agraire de Milan et par le Laboratoire Provincial Chimique de Pavie.

Dans l'ensemble, le lait obtenu pendant toute la durée de l'expérience a été de 417.818 hectolitres. Avec ce lait, on a fabriqué 130 pièces de fromage de poids uniforme et de manipulation homogène, e'est-à-dire 65 pièces avec le lait provenant des vaches du 
groupe des expériences, et 65 avec le lait provenant des vaches du groupe de contrôle. Ces pièces ont été contremarquées avec le nombre progressif de fabrication.

\section{Résultats des déterminations chimiques analytiques}

Les aliments concentrés dont on a fait l'analyse chimique ont été : le son de riz, les tourteaux de coco et d'arachide, la farine de maîs.

Les données moyennes contrôlées, comme nous l'avons déjà dit, par le Laboratoire Chimique de Pavie et par la Station Agraire Expérimentale de Milan, sont les suivantes:

\begin{tabular}{|c|c|c|c|c|c|}
\hline \multirow{2}{*}{ Eléménts considérés } & $\begin{array}{l}\text { Son de riz } \\
1^{\text {re }} \text { partie }\end{array}$ & $\begin{array}{l}\text { Son de riz } \\
2^{\mathrm{e}} \text { partie }\end{array}$ & Tourteau & Tourteau & Farine \\
\hline & $\begin{array}{c}\text { Données } \\
\text { analyti- } \\
\text { ques }\end{array}$ & $\begin{array}{c}\text { Données } \\
\text { analyti- } \\
\text { ques }\end{array}$ & d'arachide & de coco & de mais \\
\hline Humidité .......... & 7,75 & 9,10 & 6,70 & 4,26 & 9,75 \\
\hline Protéine brute ....... & 12,75 & 12,86 & 48,60 & 20,50 & 9,38 \\
\hline Matière grasse brute ... & 14,60 & 13,99 & 7,92 & 10,80 & 4,17 \\
\hline Titre adipeux-protéique & 27,35 & 26,85 & - & - & - \\
\hline
\end{tabular}

Pendant la période où l'on administra le maximum de son de riz, on exécuta quelques déterminations analytiques du beurre obtenu. Les analyses furent exécutées dans le Laboratoire Chimique de Pavie, dirigé par le Prof. N. Montr. Les résultats furent les suivants : Echantillons de la dernière semaine de février 1935.

\begin{tabular}{|c|c|c|c|}
\hline Index & $\begin{array}{l}\text { Lait de vaches } \\
\text { alimentées avec } \\
\text { son de riz à } 40 \%\end{array}$ & $\begin{array}{l}\text { Lait de vaches } \\
\text { alimentées } \\
\text { sans son de riz }\end{array}$ & $\begin{array}{c}\text { Lait mélangé de } \\
\text { toutes les vaches } \\
\text { de l'étable }\end{array}$ \\
\hline Acides volatils solubles ........ & 27,0 & 29,4 & 28,6 \\
\hline 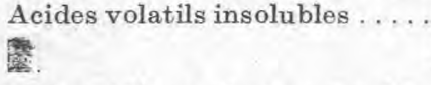 & 1,8 & 2,0 & 2,3 \\
\hline \multicolumn{4}{|c|}{ Echantillons de la première semaine de mars 1935.} \\
\hline Acides volatils solubles....... & 26,95 & 28,4 & 27,83 \\
\hline Acides volatils insolubles ..... & 1,8 & 1,8 & 2,1 \\
\hline
\end{tabular}

Les déterminations relatives aux beurres confirment les observations du Professeur Montr, au sujet de l'action réductive du son de riz sur l'index des acides volatils du beurre. Naturellement cette 
même action s'est trouvée ici moins intense qu'au cours des expériences du Professeur Monti, car évidemment elle a été en partie neutralisée, malgré l'addition de celle exercée dans le même sens par le tourteau de coco, dans la totalité de la ration alimentaire employée.

\section{Résultats zootechniques}

La Table I résume, période par période, les données des deux groupes, relativement aux quantités de lait et de beurre obtenues, ainsi que les moyennes quotidiennes de chaque animal.

Pour l'évaluation physiologique des fourrages consommés pendant toute la durée des expériences on a voulu choisir, comme terme de comparaison et de réduction, les Unités fourragères (U. F.) danoises, tenant compte toutefois de la quantité de protéine digestible contenue dans la ration alimentaire.

TABLE I.

\begin{tabular}{|c|c|c|c|c|c|c|}
\hline \multirow[b]{2}{*}{ Groupe } & \multirow[b]{2}{*}{$\begin{array}{c}\text { Nom- } \\
\text { bre } \\
\text { des } \\
\text { va- } \\
\text { ches }\end{array}$} & \multicolumn{2}{|c|}{ Lait obtenu } & \multirow[b]{2}{*}{$\begin{array}{c}\text { Pour- } \\
\text { centa- } \\
\text { ge } \\
\text { moyen } \\
\text { de ma- } \\
\text { tières } \\
\text { grasses }\end{array}$} & \multicolumn{2}{|c|}{ Beurre fabriqué } \\
\hline & & $\begin{array}{c}\text { Total } \\
\text { par période } \\
\mathrm{kg} .\end{array}$ & $\begin{array}{l}\text { Moyenne } \\
\text { quoti- } \\
\text { dienne } \\
\text { par } \\
\text { animal } \\
\mathrm{kg} \text {. }\end{array}$ & & $\begin{array}{l}\text { Total par } \\
\text { période } \\
\text { kg. }\end{array}$ & $\begin{array}{l}\text { Moyen- } \\
\text { ne quo- } \\
\text { tidienne } \\
\text { par } \\
\text { animal } \\
\text { kg. }\end{array}$ \\
\hline & \multicolumn{6}{|c|}{ 1re Période } \\
\hline Contrôle ........... & 34 & $3.486,200$ & 11,388 & 3,68 & 144,973 & 0,473 \\
\hline Expériences ....... & \multicolumn{6}{|c|}{$2^{\text {e }}$ Période } \\
\hline Contrôle .......... & 34 & $4.567,950$ & 10,430 & 3,70 & 192,836 & 0,458 \\
\hline Expériences ....... & \multicolumn{6}{|c|}{ 3e Période } \\
\hline Contrôle ......... & 34 & $5.090,600$ & 9,981 & 3,98 & 214,185 & 0,419 \\
\hline Expériences ....... & 33 & \multicolumn{5}{|c|}{$4^{\mathrm{e}}$ Période } \\
\hline Contrôle .......... & 34 & $2.727,800$ & 8,909 & 3,91 & 120,088 & 0,391 \\
\hline Expériences $\ldots \ldots \ldots$ & \multicolumn{6}{|c|}{5 e Période } \\
\hline Contrôle ......... & 34 & $4.841,300$ & 10,002 & 3,83 & 191,626 & 0,401 \\
\hline Expériences $\ldots \ldots \ldots$ & 33 & $4.959,750$ & 10,069 & 3,65 & 206,276 & 0,416 \\
\hline
\end{tabular}


La Table II indique la quantité moyenne de chaque aliment consommé pendant les différentes périodes par les animaux des deux groupes, dans la totalité et pro die pour chaque période d'expériences et dans l'ensemble pour toute la durée de l'expérience.

La Table III reproduit au contraire l'aperçu général des aliments consommés pendant les différentes périodes par les animaux des deux groupes, ainsi que le nombre des U. F. et les kilogrammes de protéine digestible contenus dans ces aliments, et déduits suivant les résultats de l'analyse chimique, à l'aide des tables expressément préparées.

La Table IV indique la valeur monétaire des différents aliments à l'époque de l'expérience, ainsi que le prix de l'U. F. et de la protéine digestible (par kilogramme) contenue dans chaque aliment.

La Table $V$ est très intéressante au point de vue des conclusions qu'on peut en tirer. Nous avons en effet indiqué, période par période et pour les deux groupes, les U. F. consommées dans l'ensemble et par chaque kilogramme de lait obtenu, les quantités de protéine digestible pareillement consommées dans l'ensemble et par chaque kilogramme de lait, le prix des U,F, et de la protéine par kilogramme.

D'après ces exposés on peut déduire comme conclusion de l'examen critique des données zootechniques réunies :

1. Que le rationnement des vaches, tel qu'il a été fait, ne peut pas être considéré strictement rationnel à cause du gaspillage qui en dérive. Cela prouve comme dans la pratique l'on poursuit encore, pour ce qui concerne l'alimentation des vaches laitières, des méthodes peu convenables, qui amènent une augmentation des frais de production, même de la part des éleveurs les mieux avisés.

2. Que la présence du son de riz dans les proportions de $25 \%$ et de $40 \%$ dans l'alimentation des vaches laitières, même pendant une assez longue période, non seulement n'altère ou ne diminue en aucune façon $l^{2}$ "appétibilité " de la ration alimentaire, mais encore le son n'est nullement nuisible à la santé de l'animal, dont l'organisme continue de fonctionner à merveille, sans manifester aucun trouble de l'appareil digestif (diarrhée, perversion du goût, etc.).

3. Que l'on n'a pas encore clairement établi l'action du son de riz sur les matières grasses du lait. D'après la Table I les grandes quantités exercent une action réductive, ce qui ne se vérifie pas pour une quantité de $25 \%$.

4. Que le son de riz, Table IV, suivant les prix de cette époque, aurait été, parmi les aliments concentrés, celui qui a donné les prix à meilleur marché des U, F. et des unités protéiques.

5. Que l'addition du son de riz au mélange alimentaire, Table V, a eu pour effet de diminuer, dans le groupe des expériences, la quan- 


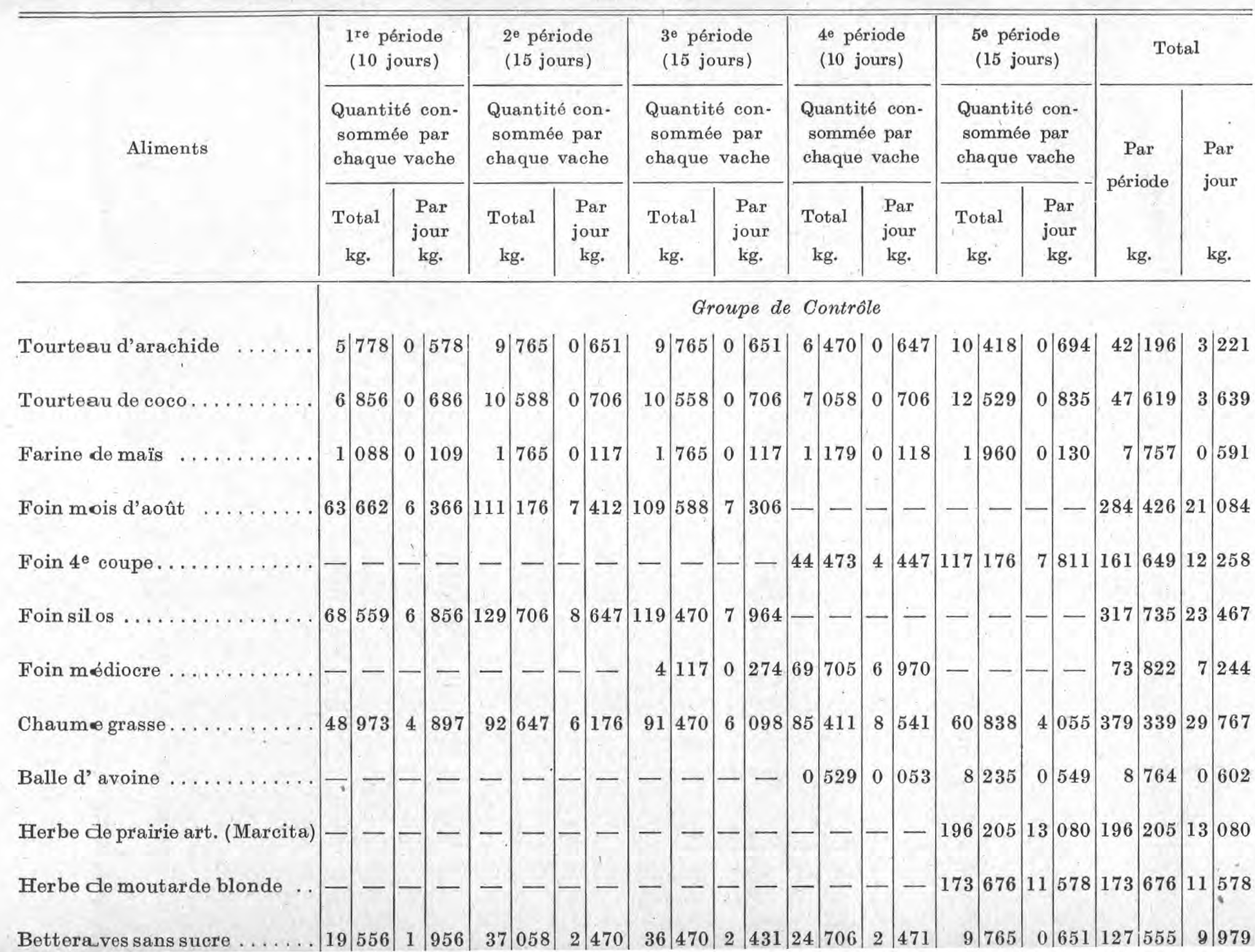




\begin{tabular}{|c|c|c|c|c|c|c|c|c|c|c|c|}
\hline \multicolumn{2}{|c|}{$\begin{array}{l}1^{\text {re }} \text { période } \\
(10 \text { jours })\end{array}$} & \multicolumn{2}{|c|}{$\begin{array}{l}2^{e} \text { période } \\
\text { ( } 15 \text { jours) }\end{array}$} & \multicolumn{2}{|c|}{$\begin{array}{l}3^{e} \text { période } \\
\text { (15 jours) }\end{array}$} & \multicolumn{2}{|c|}{$\begin{array}{l}4^{e} \text { période } \\
\text { (10 jours) }\end{array}$} & \multicolumn{2}{|c|}{$\begin{array}{l}5^{e} \text { période } \\
\text { (15 jours) }\end{array}$} & \multicolumn{2}{|c|}{ Total } \\
\hline \multicolumn{2}{|c|}{$\begin{array}{l}\text { Quantité con- } \\
\text { sommée par } \\
\text { chaque vache }\end{array}$} & \multicolumn{2}{|c|}{$\begin{array}{l}\text { Quantité con- } \\
\text { sommée par } \\
\text { chaque vache }\end{array}$} & \multicolumn{2}{|c|}{$\begin{array}{l}\text { Quantité con- } \\
\text { sommée par } \\
\text { chaque vache }\end{array}$} & \multicolumn{2}{|c|}{$\begin{array}{l}\text { Quantité con- } \\
\text { sommée par } \\
\text { chaque vache }\end{array}$} & \multicolumn{2}{|c|}{$\begin{array}{l}\text { Quantité con- } \\
\text { sommée par } \\
\text { chaque vache }\end{array}$} & \multirow{2}{*}{$\begin{array}{c}\text { Par } \\
\text { période }\end{array}$} & \multirow{2}{*}{$\begin{array}{l}\text { Par } \\
\text { jour }\end{array}$} \\
\hline $\begin{array}{l}\text { Total } \\
\text { kg. }\end{array}$ & $\begin{array}{c}\text { Par } \\
\text { jour } \\
\mathrm{kg} .\end{array}$ & Total & $\begin{array}{l}\text { Par } \\
\text { jour } \\
\text { kg. }\end{array}$ & Total & $\begin{array}{l}\text { Par } \\
\text { jour } \\
\text { kg. }\end{array}$ & $\begin{array}{c}\text { Total } \\
\mathrm{kg} .\end{array}$ & $\begin{array}{c}\text { Par } \\
\text { jour } \\
\mathrm{kg} .\end{array}$ & $\begin{array}{l}\text { Total } \\
\mathrm{kg} .\end{array}$ & $\begin{array}{l}\text { Par } \\
\text { jour } \\
\text { kg. }\end{array}$ & & \\
\hline
\end{tabular}

\begin{tabular}{|c|c|c|c|c|c|c|c|c|c|c|c|c|c|c|c|c|c|c|c|c|c|c|c|c|}
\hline & \multicolumn{24}{|c|}{ Groupe des Expériences } \\
\hline Tourteau d'arachide ... & 6 & $\mid 114$ & 0 & 611 & 7 & 500 & 0 & $500 \mid$ & 5 & $|000|$ & $0 \mid$ & $|333|$ & $|4|$ & 848 & 0 & $|485|$ & 10 & 727 & 0 & $|715|$ & 34 & 189 & 2 & 644 \\
\hline Tourteau de coco.......... & 7 & 254 & 0 & 725 & 7 & 727 & 0 & 515 & 6 & 818 & 0 & 454 & 5 & 303 & 0 & 530 & 12 & $|727|$ & 0 & 848 & 39 & 829 & 3 & \\
\hline Farine de maïs & 1 & 150 & 0 & 115 & 1 & 818 & 0 & 121 & 1 & 818 & 0 & 121 & 1 & 212 & 0 & 121 & 2 & 019 & 0 & 134 & 8 & 017 & 0 & 61 \\
\hline Son & - & - & - & - & 5 & 682 & 0 & 378 & 9 & 091 & 0 & 606 & 3 & 788 & 0 & 379 & - & - & - & - & 18 & 561 & 1 & 36 \\
\hline Foin mois d'août & 67 & 363 & 6 & 736 & 111 & 818 & 7 & 454 & 111 & 818 & 7 & 454 & - & 一 & 一 & - & - & 一 & - & - & 290 & 999 & 21 & 64 \\
\hline Foin & - & - & - & - & - & 一 & - & - & - & - & - & - & 44 & 727 & 4 & 473 & 120 & 727 & 8 & 048 & 165 & 454 & 12 & 52 \\
\hline Foin silos & 72 & 545 & 7 & 254 & 172 & 878 & 11 & 525 & 121 & 757 & 8 & 117 & - & - & - & - & 一 & 一 & - & - & 367 & 180 & 26 & 89 \\
\hline Foir & - & 一 & - & - & - & - & - & - & 4 & 151 & 0 & 276 & 69 & 636 & 6 & 964 & - & - & - & - & 73 & 787 & 7 & 24 \\
\hline Chaume grasse.... & 51 & 818 & 5 & 182 & 93 & 181 & 6 & 212 & 93 & 181 & 6 & 212 & 64 & 060 & 6 & 406 & 62 & 681 & 4 & 178 & 364 & 921 & 28 & 190 \\
\hline Balle d & - & - & - & - & - & - & - & - & - & - & - & - & 0 & 545 & 0 & 055 & 8 & 727 & 0 & 582 & 9 & 272 & 0 & 63 \\
\hline Herbe de parairie art. (Marcita) & - & - & - & - & - & - & - & - & - & - & - & - & - & - & - & - & 202 & 150 & 13 & 476 & 202 & 150 & 13 & 47 \\
\hline Herbe de moutarde blanche.. & - & - & - & - & - & - & - & - & - & - & - & - & $\therefore$ & 一 & - & - & 178 & 939 & 11 & 929 & 178 & 939 & 11 & 92 \\
\hline & $?$ & $|79.7|$ & 9 & $|073|$ & $\mathbf{3}$ & $|272|$ & 2 & $|484|$ & 37 & $|272|$ & $|2|$ & $|484|$ & $|24|$ & 848 & 2 & $|485|$ & 10 & $|060|$ & 0 & $|670|$ & $|130|$ & 179 & $|10|$ & \\
\hline
\end{tabular}


TABLE III.

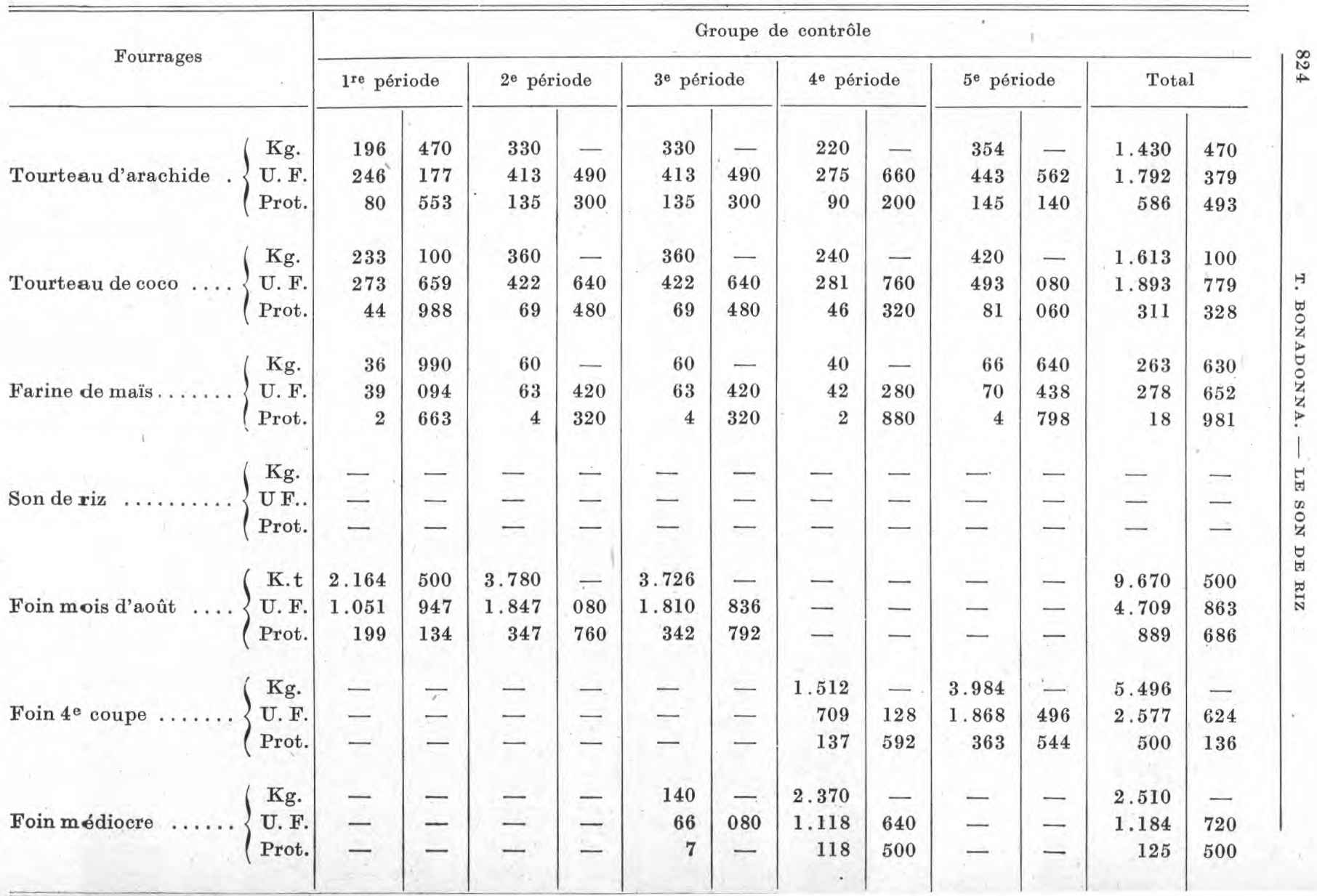




\begin{tabular}{|c|c|c|c|c|c|c|c|c|c|c|c|c|c|}
\hline \multirow{2}{*}{\multicolumn{2}{|c|}{ Fourrages }} & \multicolumn{12}{|c|}{ Groupe des expériences } \\
\hline & & \multicolumn{2}{|c|}{$1^{\text {re }}$ période } & \multicolumn{2}{|c|}{$2^{\mathrm{e}}$ période } & \multicolumn{2}{|c|}{$3^{\mathrm{e}}$ période } & \multicolumn{2}{|c|}{$4^{\mathrm{e}}$ période } & \multicolumn{2}{|c|}{$5^{e}$ période } & \multicolumn{2}{|c|}{ Total } \\
\hline . & $\mathrm{Kg}$. & 2.331 & - & 4.410 & - & 4.060 & - & - & - & - & - & 10.801 & - \\
\hline Foin silos & U. F. & 512 & 820 & 970 & 200 & 893 & 200 & - & - & - & - & 2.376 & 220 \\
\hline & Prot. & 44 & 289 & 83 & 790 & 77 & 140 & 一 & - & - & - & 205 & 219 \\
\hline & $\mathrm{Kg}$. & 666 & - & 1.260 & - & 1.240 & - & 840 & - & 332 & - & 4.338 & - \\
\hline Betteraves sans sucre & U. F. & 85 & 914 & 162 & 540 & 159 & 960 & 108 & 630 & 42 & 828 & 559 & 872 \\
\hline & Prot. & 7 & 992 & 15 & 120 & 14 & 880 & 10 & 080 & 3 & 984 & 52 & 056 \\
\hline & $\mathrm{Kg}$. & 1.665 & - & 3.150 & - & 3.110 & - & 2.904 & - & 2.068 & 500 & 12.897 & 500 \\
\hline Chaume grasse & U. F. & 391 & 275 & 740 & 250 & 730 & 850 & 682 & 440 & 486 & 097 & 3.030 & 912 \\
\hline & Prot. & 21 & 645 & 40 & 950 & 40 & 430 & 37 & 752 & 26 & 891 & 167 & 668 \\
\hline & Kg. & - & - & - & - & - & - & 18 & - & 288 & - & 306 & 一 \\
\hline Balle d'avoine & U. F. & - & - & - & - & - & - & 7 & 020 & 112 & 320 & 119 & 340 \\
\hline & Prot. & - & - & - & - & - & - & 0 & 361 & 5 & 472 & 7 & 883 \\
\hline Herbe de prairie artifi- & $\mathrm{Kg}$. & - & - & - & - & - & - & - & - & 6.671 & - & 6.671 & - \\
\hline Herbe de prairie artifi- & U. F. & - & - & - & - & - & - & - & - & 773 & 836 & 773 & 836 \\
\hline cielle (Marcita) ... & Prot. & - & - & - & - & 一 & - & - & - & 73 & 381 & 73 & 381 \\
\hline & $\mathrm{Kg}$. & - & - & - & - & - & - & - & - & 5.905 & - & 5.905 & - \\
\hline blanche & U.F. & 一 & - & - & - & - & - & - & - & 590 & 500 & 590 & 500 \\
\hline & Prot. & 一 & - & - & - & - & - & - & - & 112 & 190 & 112 & 190 \\
\hline & U. F. & 2.600 & 886 & 4.619 & 620 & 4.560 & 476 & 3.225 & 559 & 4.881 & 157 & 19.887 & 698 \\
\hline & Prot. & 410 & 264 & 696 & 720 & 691 & 342 & 443 & 685 & 815 & 460 & 3.057 & 471 \\
\hline
\end{tabular}


TABLE III (Suite).

\begin{tabular}{|c|c|c|c|c|c|c|c|c|c|c|c|c|c|}
\hline \multirow{2}{*}{ Fourrages } & & \multicolumn{12}{|c|}{ Groupe de contrôle } \\
\hline & & \multicolumn{2}{|c|}{$1^{\text {re }}$ période } & \multicolumn{2}{|c|}{$2^{\mathrm{e}}$ période } & \multicolumn{2}{|c|}{$3^{e}$ période } & \multicolumn{2}{|c|}{$4^{e}$ période } & \multicolumn{2}{|c|}{$5^{\text {e }}$ période } & \multicolumn{2}{|c|}{ Total } \\
\hline \multirow{3}{*}{ Tourteau d'arachide } & $\mathbf{K g}$. & 201 & 780 & 247 & 500 & 165 & - & 160 & - & 354 & - & 1.112 & 280 \\
\hline & U. F. & 252 & 830 & 310 & 117 & 206 & 745 & 200 & 480 & 443 & 562 & 1.413 & 734 \\
\hline & Prot. & 82 & 730 & 101 & 475 & 67 & 650 & 65 & 600 & 145 & 140 & , 462 & 595 \\
\hline \multirow{3}{*}{ Tourteau de coco } & $\mathrm{Kg}$. & 239 & 400 & 255 & 一 & 225 & - & 175 & $\longrightarrow$ & 420 & - & 1,314 & 400 \\
\hline & U. F. & 281 & 056 & 299 & 370 & 264 & 150 & 205 & 450 & 493 & 080 & 1.543 & 160 \\
\hline & Prot. & 46 & 204 & 49 & 215 & 43 & 425 & 33 & 775 & 81 & 060 & 253 & 679 \\
\hline \multirow{3}{*}{ Farine de mais. } & $\mathrm{Kg}$. & 37 & 980 & 60 & - & 60 & - & 40 & - & 66 & 640 & 264 & 620 \\
\hline & U. F. & 40 & 145 & 63 & 420 & 63 & 420 & 42 & 280 & 70 & 438 & 279 & 703 \\
\hline & Prot. & 2 & 735 & 4 & 320 & 4 & 320 & 2 & 880 & -4 & 798 & 19 & 053 \\
\hline \multirow{3}{*}{ Son de riz } & $\mathrm{Kg}$. & - & - & 187 & 500 & 300 & - & 125 & - & - & 一 & 612 & 500 \\
\hline & U. F. & - & - & 159 & 375 & 255 & - & 106 & 250 & - & 一 & 520 & 625 \\
\hline & Prot. & - & - & 14 & 625 & 23 & 400 & 9 & 750 & - & - & 47 & 775 \\
\hline \multirow{3}{*}{ Foin mois d'août } & $\mathrm{Kg}$. & 2.223 & - & 3.690 & - & 3.690 & - & - & 一 & - & - & 9.603 & - \\
\hline & U. F. & 1.080 & 378 & 1.793 & 340 & 1.793 & 340 & - & - & - & - & 4.667 & 058 \\
\hline & Prot. & 204 & 516 & 339 & 480 & 339 & 480 & - & - & - & - & 883 & 476 \\
\hline \multirow{3}{*}{ Foin $4^{\mathrm{e}}$ coupe . } & $\mathrm{Kg}$. & - & - & - & - & - & - & 1.476 & - & 3.984 & - & 5.460 & - \\
\hline & U. F, & - & - & - & - & - & - & 692 & 294 & 1. 868 & 496 & 2.560 & 740 \\
\hline & Prot. & - & - & - & - & - & - & 134 & 316 & 362 & 544 & 496 & 860 \\
\hline \multirow{3}{*}{ Foin médiocre. } & $\mathbf{K g}$. & - & - & - & - & 137 & - & 2.298 & - & - & - & 2.435 & - \\
\hline & U. F. & - & - & - & - & 64 & 664 & 1.084 & 656 & - & - & 1.149 & 320 \\
\hline & Prot. & - & - & - & - & 6 & 850 & 114 & 900 & - & - & 121 & 750 \\
\hline
\end{tabular}


TABLE III (Fin).

\begin{tabular}{|c|c|c|c|c|c|c|c|c|c|c|c|c|c|}
\hline \multirow{2}{*}{ Fourrages } & & \multicolumn{12}{|c|}{ Groupe des expériences } \\
\hline & & \multicolumn{2}{|c|}{$1^{\text {re }}$ période } & \multicolumn{2}{|c|}{$2^{e}$ période } & \multicolumn{2}{|c|}{$3^{e}$ période } & \multicolumn{2}{|c|}{$4^{e}$ période } & \multicolumn{2}{|c|}{$5^{e}$ période } & \multicolumn{2}{|c|}{ Total } \\
\hline & $\mathrm{Kg}$. & 2.394 & 一 & 4.305 & - & 4.018 & - & - & - & - & - & 1.717 & - \\
\hline Foin silos . & U. F. & 526 & 680 & 947 & 100 & 883 & 960 & - & - & - & - & 2.357 & 740 \\
\hline & Prot. & 45 & 486 & 81 & 795 & 76 & 342 & - & - & - & - & 203 & 623 \\
\hline$\cdot$ & $\mathrm{Kg}$. & 684 & $T$ & 1.230 & - & 1.230 & - & 820 & - & 332 & - & 4.296 & 一 \\
\hline Betteraves sans sucre. & U. F. & 88 & 236 & 158 & 670 & 158 & 670 & 105 & 780 & 42 & 828 & 554 & 184 \\
\hline 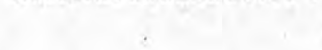 & Prot. & 8 & 206 & 14 & 760 & 14 & 760 & 9 & 840 & 3 & 984 & 51 & 552 \\
\hline & Kg. & 1.710 & - & 3.075 & - & 3.075 & - & 2.014 & - & 2.068 & 500 & 11.942 & 500 \\
\hline Chaume grasse . & U. F. & 401 & 850 & 722 & 625 & 722 & 625 & 472 & 290 & 486 & 097 & 2.806 & 487 \\
\hline . & Prot. & 22 & 230 & 39 & 975 & 39 & 975 & 26 & 182 & 26 & 891 & 155 & 253 \\
\hline & $\mathrm{Kg}$. & - & - & - & - & - & - & 18 & - & 288 & 一 & 306 & - \\
\hline Balle d'avoine ....... & U. F. & 一 & - & - & - & - & - & 7 & 020 & 112 & 320 & 119 & 340 \\
\hline & Prot. & - & - & 一 & - & - & - & 0 & 361 & 5 & 472 & 5 & 833 \\
\hline Honbedonntifi & $\mathrm{Kg}$. & - & - & - & - & - & - & - & 一 & 6.671 & - & 6.671 & - \\
\hline Herbe de prairie artifi- & U. F. & - & - & - & - & - & 一 & - & - & 773 & 836 & 773 & 836 \\
\hline cielle (Marcita) ... & Prot. & - & - & 一 & - & - & - & - & - & 73 & 381 & 73 & 381 \\
\hline 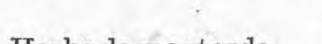 & $\mathrm{Kg}$. & - & - & - & 一 & - & - & - & - & 5.905 & - & 5.905 & 一 \\
\hline Herbe de moutarde & U. F. & - & - & - & - & - & - & - & - & 590 & 500 & 590 & 500 \\
\hline blanche ..... & Prot. & - & - & - & - & - & - & - & - & 112 & 195 & 112 & 195 \\
\hline & U. F. & 2.671 & 535 & 4.454 & 017 & 4.412 & 574 & 2.917 & 450 & 4.881 & 157 & 19.336 & 733 \\
\hline & Prot. & 412 & 109 & 645 & 655 & 616 & 212 & 397 & 604 & 815 & 465 & 2.887 & 045 \\
\hline
\end{tabular}




\begin{tabular}{|c|c|c|c|c|c|c|}
\hline Fourrage & & 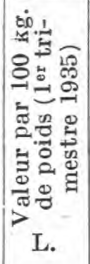 & $\begin{array}{c}\begin{array}{c}\text { Quantité } \\
\text { consommée }\end{array} \\
\text { L. }\end{array}$ & $\begin{array}{c}\text { Prix total } \\
\text { L. }\end{array}$ & 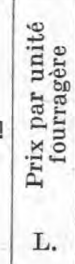 & $\begin{array}{c}\text { Prix } \\
\text { par kg. } \\
\text { de pro- } \\
\text { téine } \\
\text { digesti. } \\
\text { ble } \\
\text { L. }\end{array}$ \\
\hline Tourteau d'arachide & $\begin{array}{l}\text { Contrôle } \\
\text { Expériences }\end{array}$ & 44 & $\begin{array}{l}1.430,470 \\
1.128,280\end{array}$ & $\begin{array}{l}630,40 \\
496,45\end{array}$ & $\begin{array}{l}0,90 \\
0,90\end{array}$ & $\begin{array}{l}4,79 \\
4,78\end{array}$ \\
\hline Tourteau de coco . & $\begin{array}{l}\text { Contrôle } \\
\text { Expériences }\end{array}$ & 52 & $\begin{array}{l}1.613,100 \\
1.314,400\end{array}$ & $\begin{array}{l}838,80 \\
683,50\end{array}$ & $\begin{array}{l}1,06 \\
1,06\end{array}$ & $\begin{array}{l}5,65 \\
5,48\end{array}$ \\
\hline Farine de maïs . . & $\begin{array}{l}\text { Contrôle } \\
\text { Expériences }\end{array}$ & 65 & $\begin{array}{l}263,630 \\
264,620\end{array}$ & $\begin{array}{l}171,35 \\
172,00\end{array}$ & $\begin{array}{l}1,33 \\
1,33\end{array}$ & $\begin{array}{l}7,07 \\
7,07\end{array}$ \\
\hline Son de riz $\ldots \ldots$ & $\begin{array}{l}\text { Contrôle } \\
\text { Expériences }\end{array}$ & 33,50 & 612,500 & 205,20 & 0,62 & 3,64 \\
\hline Foin mois d'août. & $\begin{array}{l}\text { Contrôle } \\
\text { Expériences }\end{array}$ & 20 & $\begin{array}{l}9.670,500 \\
9.603,--\end{array}$ & $\begin{array}{l}1.934,10 \\
1.9 \div 0,60\end{array}$ & $\begin{array}{l}0,40 \\
0,40\end{array}$ & $\begin{array}{l}2,17 \\
2,17\end{array}$ \\
\hline Foin $4^{\mathrm{e}}$ coupe. & $\begin{array}{l}\text { Contrôle } \\
\text { Expériences }\end{array}$ & 14 & $\begin{array}{l}5.496,-- \\
5.460,-\end{array}$ & $\begin{array}{r}769,45 \\
764,40\end{array}$ & $\begin{array}{l}0,28 \\
0,29\end{array}$ & $\begin{array}{l}1,52 \\
1,52\end{array}$ \\
\hline Foin médiocre & $\begin{array}{l}\text { Contrôle } \\
\text { Expériences }\end{array}$ & 12 & $\begin{array}{l}2.510, \\
2.435\end{array}$ & $\begin{array}{l}301,20 \\
292,20\end{array}$ & $\begin{array}{l}0,24 \\
0,24\end{array}$ & $\begin{array}{l}1,30 \\
1,30\end{array}$ \\
\hline Foin silos . . & $\begin{array}{l}\text { Contrôle } \\
\text { Expériences }\end{array}$ & 10 & $\begin{array}{l}10.801,-- \\
10.717,--\end{array}$ & $\begin{array}{l}1.080,10 \\
1.071,70\end{array}$ & $\begin{array}{l}0,20 \\
0,20\end{array}$ & $\begin{array}{l}1,08 \\
1,08\end{array}$ \\
\hline Betteraves s. sucre & $\begin{array}{l}\text { Contrôle } \\
\text { Expériences }\end{array}$ & 9 & $\begin{array}{l}4.338,-- \\
4.296 .--\end{array}$ & $\begin{array}{l}390,40 \\
385,65\end{array}$ & $\begin{array}{l}0,18 \\
0,18\end{array}$ & $\begin{array}{l}0,98 \\
0,98\end{array}$ \\
\hline Chaume grasse. & $\begin{array}{l}\text { Contrôle } \\
\text { Expériences }\end{array}$ & 9 & $\begin{array}{l}12.897,500 \\
11.942,500\end{array}$ & $\begin{array}{l}1.160,75 \\
1.074,80\end{array} \mid$ & $\begin{array}{l}0,18 \\
0,18\end{array}$ & $\begin{array}{l}0,97 \\
0,97\end{array}$ \\
\hline Balle d'avoine $\ldots .$. & $\begin{array}{l}\text { Contrôle } \\
\text { Expériences }\end{array}$ & 9 & $\begin{array}{l}206,- \\
306,-\end{array}$ & $\begin{array}{l}185,40 \\
275,40\end{array}$ & $\begin{array}{l}1,84 \\
1,84\end{array}$ & $\begin{array}{l}9,72 \\
9,77\end{array}$ \\
\hline $\begin{array}{l}\text { Herbe de prairie arti- } \\
\quad \text { ficielle (Marcita) .. }\end{array}$ & $\begin{array}{l}\text { Contrôle } \\
\text { Expériences }\end{array}$ & 5 & $\begin{array}{l}6.671,- \\
6.671,-\end{array}$ & $\begin{array}{l}333,55 \\
333,55\end{array}$ & $\begin{array}{l}0,10 \\
0,10\end{array}$ & $\begin{array}{l}0,54 \\
0,54\end{array}$ \\
\hline $\begin{array}{l}\text { Herbe de moutarde } \\
\text { blanche } \ldots \ldots \ldots\end{array}$ & $\begin{array}{l}\text { Contrôle } \\
\text { Expériences }\end{array}$ & 4 & $\begin{array}{l}5.905,-- \\
5.905,-\end{array}$ & $\begin{array}{l}236,20 \\
236,20\end{array}$ & $\begin{array}{l}0,08 \\
0,08\end{array}$ & $\begin{array}{l}0,43 \\
0,43\end{array}$ \\
\hline
\end{tabular}


TABLE $V$.

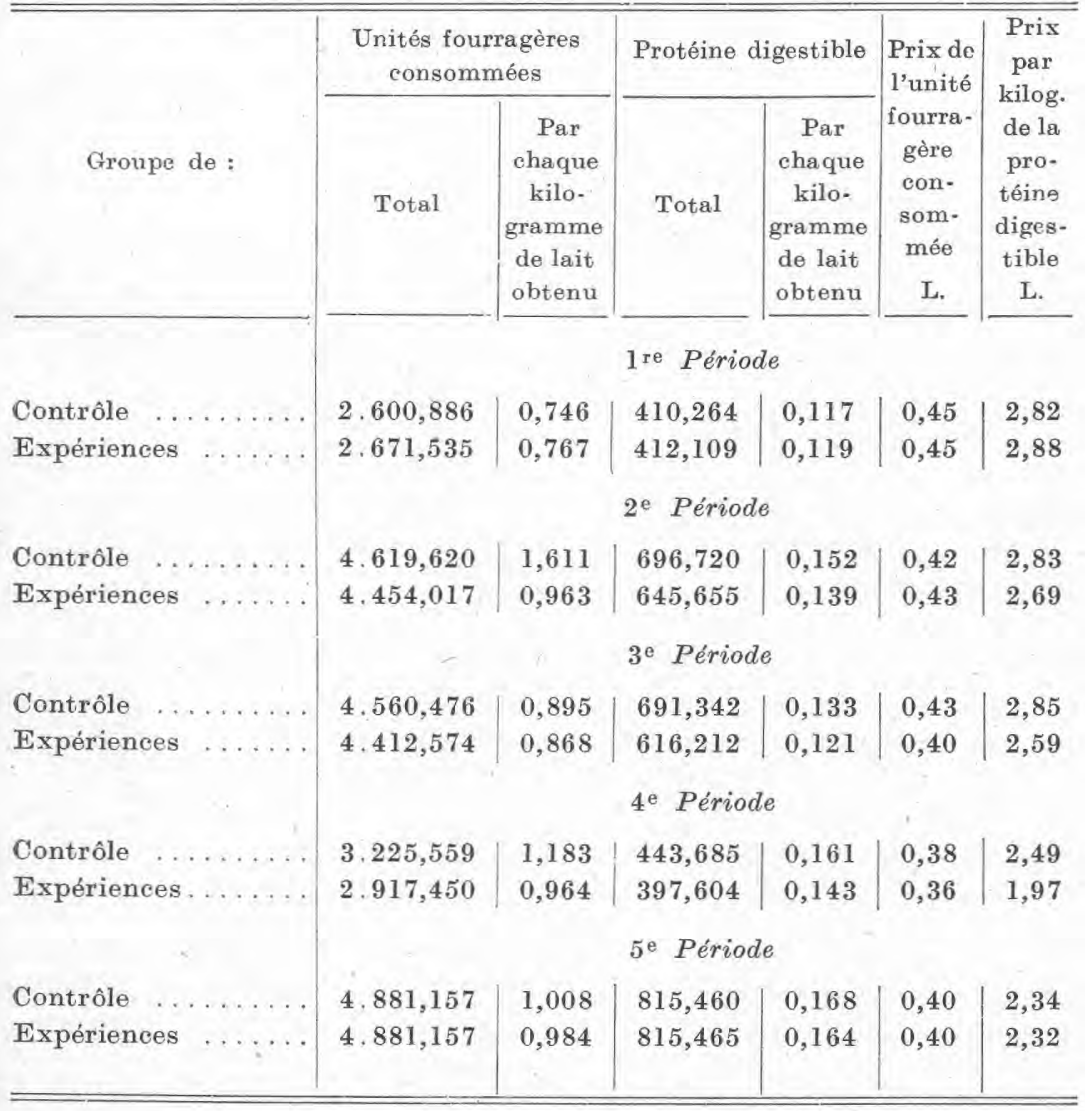

tité des U. F. nécessaires à la production d'un litre de lait, ainsi que la quantité de protéine digestible consommée pour chaque kilogramme de lait obtenu. Que dans le groupe des expériences le prix des U. F. consommées a été inférieur à celui du groupe de contrôle pendant la troisième et la quatrième période, et quelque peu supérieur seulement pendant la deuxième période, tandis que le prix de l'unité protéique a été inférieur pendant la deuxième, troisième et quatrième période de l'expérience.

6. Qu'enfin, d'après la Table VI, on peut déduire que le prix par kilogramme de lait, dans le groupe des expériences, a été inférieur pendant la deuxième et la troisième période et puis encore pendant la quatrième période, et que la quantité des U. F. et des protéines digestibles nécessaires pour obtenir un litre de lait, a toujours été décidément inférieure pendant les trois périodes des expériences.

7. Que pour conclure, du point de vue purement zootechnique 
TABLE VI.

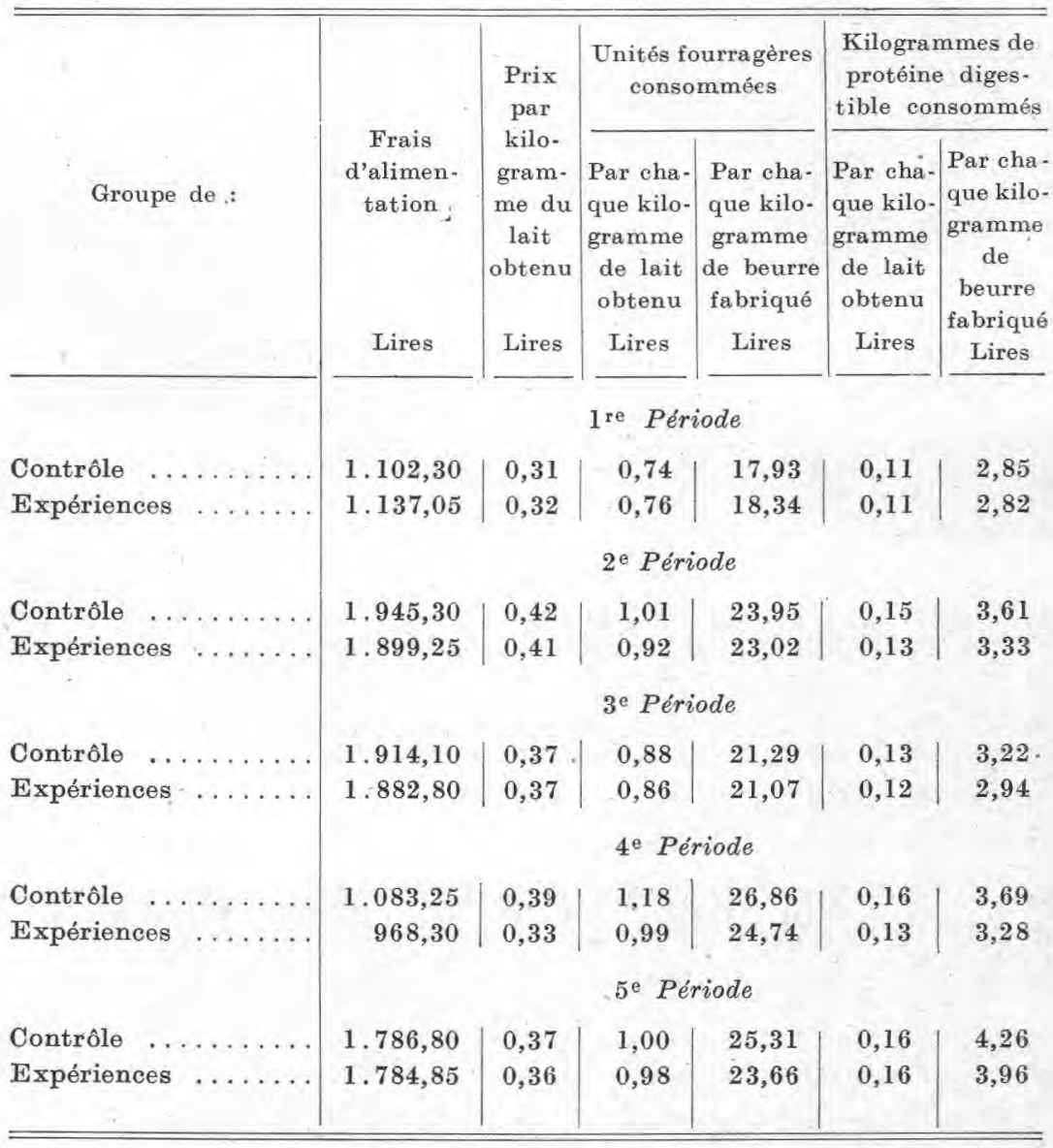

et suivant les prix des différents aliments consommés pendant le premier trimestre de 1935 , on peut affirmer que l'emploi du son de riz, même dans le maximum de quantité pareille à $40 \%$, est recommandable pour l'alimentation des vaches laitières, d'abord parce qu'il n'est pas nuisible à la santé des animaux, et ensuite en raison du bénéfice économique qui peut être réalisé en vue de la diminution des frais de production. Il faut, bien entendu, que le son employé soit toujours de bonne qualité, pur et bien conservé.

8. Qu'il est indispensable que les recherches commencées soient ultérieurement poursuivies, dans le but d'établir la limite du maximum de quantité de son de riz qui peut être employé dans l'alimentation des vaches laitières, avec tolérance de la part de l'organisme et réel avantage économique; la quantité optimum de 
son de riz qui peut être employée sans provoquer des diminutions dans le taux butyreux du lait ; la quantité de son de riz qui peut être convenablement employée pendant les différentes saisons de l'année, et les conséquences qui peuvent s'ensuivre pour ce qui concerne la réussite des différents produits caséeux; le genre des aliments avec lesquels le son de riz peut être convenablement mélangé pour compenser les insuffisances nutritives ainsi que l'éventuelle action anticaséeuse, comme on verra ensuite.

\section{Résultats " fromagers »}

Comme nous l'avons déjà dit, un jugement définitif à propos des résultats fromagers serait tout à fait prématuré.

Il faut en effet attendre les résultats de la maturation complète des pièces de fromage fabriquées, et qui se trouvent actuellement à la fromagerie de la ferme Spizzi. L'évaluation effective de la réussite des fromages devra ensuite être complétée par l'examen des caractères fondamentaux, tant typiques qu'intrinsèques.

Cela paraît surtout nécessaire comme déduction logique des observations révélatrices faites par le Professeur Costantino GoRINI, au cours de l'expérience pendant le mois de mars. Le Professeur Gorin, interpellé par MM. SpIzzi, pour établir les causes des phénomènes fromagers anormaux qui s'étaient vérifiés vers la fin de l'expérience, et précisément lorsqu'on supprima l'administration du son de riz, en le remplaçant par d'autres aliments, put donner les indices des inconvénients fromagers causés par la balle d'avoine qui est aussi largement employée dans la région lombarde.

Les recherches terminées dès lors et celles en cours avec nos nouvelles recherches, ont mis en rapport l'action de certains fourrages, avec la sécrétion de ce que le Professeur Gokini a défini "Lait 'disgenesico' " (1).

De là, notre décision d'approfondir nos observations, suivant un plan d'expériences plus complexe, e'est-à-dire en étendant les recherches à tous les fourrages employés, tant singulièrement que dans de différents mélanges alimentaires, car nous désirons préciser l'action spécifique et les limites de rapport zootechniques et fromagers de leur emploi, faisant valoir pour la première fois leur action éventuelle sur les caractères typiques et intrinsèques des fromages fabriqués.

De toutes nos recherches, comme de toutes les expériences faites dans le champ pratique de l'alimentation, il ressort combien sont nombreux les facteurs qui, même pour ce qui concerne la ration

(1) C. GoRINI. Foraggi anticaseari e latte a disgenesico ", Reale Istituto Lombardo di Scienze e Lettere, 1935 (Fourrages anti-fromagers et lait "disgenesico", Comptes rendus du Royal Institut Lombard de Sciences et Lettres, 1935). 
alimentaire, concourent à rendre la production du lait, non seulement plus ou moins coûteuse et plus ou moins bienfaisante pour le bétail, mais encore à modifier profondément la flore bactérienne du lait (d'après les recherches du Professeur GoRini dans le lait " disgenesico ") en obtenant des effets remarquables par rapport au traitement du lait et aux résultats des produits fromagers.

On peut donc déduire la nécessité d'ordre spéculatif et d'ordre pratique d'orienter les recherches vers le champ d'alimentation des vaches laitières, de façon à pouvoir identifier l'action fromagère ou anti-fromagère des aliments les plus communs, afin d'établir leur association convenable zooéconomique, et pour exploiter et équilibrer les actions antagonistes et compensatrices.

Pour finir, nous dirons que pour ce qui concerne les résultats de l'expérience susdite, ainsi que leur exposition et leur discussion, on est contraint pour l'instant de les ajourner. Ces résultats seront recueillis dans un futur article, que nous nous réservons de préparer dès qu'il nous sera possible.

Pourtant on a commencé en 1936 d'autres expériences sur l'alimentation du bétail et sur le contrôle fromager, expériences quî sont actuellement en cours et qui comprennent un champ d'action beaucoup plus vaste que le précédent.

Pavie, 5 mars 1936.

\title{
QUELQUES OBSERVATIONS CONCERNANT L'ACTION DE LA SOLUTION DE CHLORURE DE CALCIUIM POUR RÉFRACTOMÉTRIE SUR LES LAITS ADDITIONNÉS DE CARBONATES ALCALINS
}

\author{
par \\ A. TAPERNOUX \\ Professeur de Chimie \\ à l'Ecole Vétérinaire de Lyon
}

Une très longue pratique de la réfractométrie appliquée au lait, en utilisant la méthode de préparation du sérum chlorocalcique, selon Ackermann [1, 2], m'a permis de faire l'observation suivante: les laits non altérés par la fermentation lactique ou par un trouble de la sécrétion, mais qui ont été additionnés de carbonates ou de bicarbonates alcalins, coagulent mal ou ne coagulent pas du tout au cours des manipulations employées pour préparer le sérum. Si la dose de sel alcalin ajoutée au lait est faible, la coagulation se produit et la synérèse laisse sourdre un sérum trouble; si la dose de sel alcalin est plus forte, le mélange lait + solution de chlorure de calcium 